\title{
QuESTIONÁRIOS SOBRE O USO DE ÁlCOOL E DROGAS ENTRE TRABALHADORES: REVISÃO DA LITERATURA
}

\section{Patrícia Rodrigues da Rocha ${ }^{1}$; Helena Maria Scherlowski Leal David ${ }^{2}$}

A questão do uso de álcool e drogas tem sido crescentemente reconhecida como problema para a saúde dos trabalhadores, apesar dos desafios metodológicos para este tipo de pesquisa. Trata-se de estudo de revisão bibliográfica com o objetivo de identificar e analisar publicações que apresentam enfoque no uso de álcool e drogas por trabalhadores, com foco nos instrumentos de coleta de dados. A revisão abrangeu o período de 1998 a 2008. A metodologia contemplou a definição e aplicação de descritores, e a busca em bases de dados digitalizadas. Após leitura dos resumos e aplicados os critérios de exclusão, foram selecionadas 33 publicações para análise. Os resultados evidenciaram que os questionários Cage e Audit foram os mais utilizados nas pesquisas, e a maioria dos trabalhadores estudados pertencia ao setor de serviços, com poucos estudos nos demais setores.

Descritores: Alcoolismo; Enfermagem; Questionários; Saúde do Trabalhador.

\footnotetext{
${ }^{1}$ Enfermeira, Mestranda em Enfermagem, Faculdade de Enfermagem, Universidade do Estado do Rio de Janeiro, RJ, Brasil. E-mail: patriciarodrigues0@yahoo.com.br.

${ }^{2}$ Enfermeira, Doutor em Saúde Pública, Professor Adjunto, Faculdade de Enfermagem, Universidade do Estado do Rio de Janeiro, RJ, Brasil. E-mail: helena.david@oi.com.br.
}

Endereço para Correspondência

Patrícia Rodrigues da Rocha

Universidade Federal Rural do Rio de Janeiro

Núcleo de Atenção à Saúde do Servidor da Universidade Rural

BR 465, Km 07

CEP: 23890-000, Seropédica, RJ, Brasil

E-mail: patriciarodrigues0@yahoo.com.br 


\section{Alcohol And Drugs QUestionnaire AMONG WORKERS: A LITERATURE}

\section{REVIEW}

Alcohol and drug use has been increasingly recognized as an occupational health problem, despite methodological difficulties to accomplish this type or research. A bibliographic review was carried out to identify and analyze papers that have focused on workers ' use of alcohol and drugs, focusing on data collection instruments. The review period was from 1998 to 2008. The method included the definition of descriptors and the search in on-line databases. After abstract reading and exclusion criteria use, 33 papers were selected for analysis. Results showed that CAGE and AUDIT questionnaires were the most frequently used, and that the majority of studied workers were from service sector, with few studies in other sectors.

Descriptors: Alcoholism; Nursing; Questionnaires; Occupational Health.

\section{Cuestionarios SObRe EL USO DE ALCOHOL Y DROGAS ENTRE LOS TRABAJADORES: REVISIÓN DE LA LITERATURA}

El uso de alcohol y drogas es reconocido como un problema para la salud de los trabajadores, a pesar de las dificultades metodológicas para ese tipo de estudio. Se desarrolló una revisión bibliográfica para identificar y analizar publicaciones que se han centrado en el consumo de alcohol y drogas por trabajadores, con foco en los instrumentos de recolección de datos. La revisión incluyo el periodo desde 1998 hasta 2008. Después de la lectura de los resúmenes y la aplicación de criterios de exclusión, 33 publicaciones fueran seleccionadas para análisis. Los resultados muestran que los cuestionarios CAGE y AUDIT fueran los más usados, y que la mayoría de los trabajadores estudiados estaban en el sector de servicios, con pocos estudios en otros sectores.

Descriptores: Alcoholismo; Enfermería; Cuestionario; Salud Laboral.

\section{Introdução}

O relatório das Nações Unidas sobre As Tendências Mundiais das Drogas Ilícitas, publicado em 2003, retrata que 200 milhões de pessoas no mundo, aproximadamente, consumiram alguma droga ilícita entre 2000 e $2001^{(1)}$.

Pesquisa promovida pela Secretaria Nacional de Políticas sobre Drogas (Senad) aponta que, de uma população adulta de 120 milhões, 27,6 milhões têm ou já podem ter tido algum problema relacionado ao uso do álcool. Homens e pessoas mais jovens apresentam maiores taxas de problemas em relação às mulheres e mais velhos ${ }^{(2)}$.

$\mathrm{O}$ uso e abuso de drogas lícitas e ilícitas, principalmente o álcool, por trabalhadores, tem se constituído em preocupação por parte das empresas, em função do prejuízo observado na produção ${ }^{(3)}$.

$\mathrm{O}$ estudo $^{(4)}$ realizado no município do Rio de Janeiro, em 2002, revelou que, entre os possíveis riscos para o uso e abuso de drogas, está o estresse gerado pelo trabalho, a rivalidade com colegas, problemas pessoais e insatisfação com o trabalho e função que desempenham.

A compreensão sobre as relações entre trabalho e uso de drogas, entretanto, ainda é insuficiente no Brasil ${ }^{(5)}$. Isso pode ser também afirmado em relação a outros países, que descrevem a relação entre drogas e violência no mundo do trabalho, concluindo que há subregistro a respeito do 
problema, contrapondo-se a dados norte-americanos, que acusam aumento de $300 \%$ da violência laboral, com base no aumento do consumo de álcool pelos trabalhadores ${ }^{(6)}$.

Este artigo pretende colaborar para a ampliação do conhecimento acerca dos instrumentos utilizados para avaliar o padrão de consumo de álcool e outras drogas entre trabalhadores, a partir da identificação da insuficiência de questionários autoaplicáveis para essa finalidade, principalmente, em relação às estratégias utilizadas pelas empresas para promoção e prevenção do fenômeno das drogas entre os trabalhadores. A proposta de um instrumento de coleta de dados autoaplicável está intimamente relacionada ao fato de se manter o sigilo e o anonimato do trabalhador, levando-os a se sentirem, como sujeitos da pesquisa, mais protegidos para responder ao questionário, evidenciando-se como questão metodológica importante para a obtenção de dados mais consistentes.

Os objetivos deste estudo foram: levantar os estudos publicados, que apresentam enfoque no uso de álcool e drogas por trabalhadores, entre os anos 1998 e 2008, identificando e categorizando os instrumentos de coleta de dados utilizados nos estudos; analisar os estudos relacionados diretamente à avaliação do padrão de consumo de álcool e outras drogas, através da aplicação de um questionário.

\section{Material e Métodos}

Trata-se de estudo de revisão bibliográfica, realizada em bases de dados disponíveis na internet. Foram utilizados os descritores cadastrados no sistema Descritores em Ciências da Saúde (DeCS), adotados pela BIREME. Os descritores utilizados foram: alcoolismo, bebidas alcoólicas, drogas ilícitas, trabalho, trabalhadores $e$ questionários. Acrescentou-se à busca palavras-chave de significado próximo ao descritor na intenção de encontrar estudos que poderiam não estar utilizando descritores padronizados (abuso de álcool, abuso de drogas e drogas de abuso).

As bases de dados acessadas foram: Banco de Teses da Capes - Coordenação de Aperfeiçoamento de Pessoal de Nível Superior e BVS - Biblioteca Virtual em Saúde. A BVS é composta por diversos bancos de dados, dos quais foram utilizados: BDENF - Base de Dados de Enfermagem, SciELO - Scientific Electronic Library Online, LILACS - Literatura Latino-Americana e do Caribe em Ciências da Saúde, e MEDLINE - Literatura Internacional em Ciências da Saúde.

A partir da leitura dos resumos, os estudos foram selecionados de acordo com os seguintes critérios de inclusão: possuir enfoque no estudo do uso de bebidas alcoólicas e outras drogas entre trabalhadores, ter como instrumento de coleta de dados um questionário, a população ou amostra do estudo ser constituída por trabalhadores, estudos publicados entre 1998 e 2008, estudos quantitativos ou quantiqualitativos, estudos publicados em português, inglês ou espanhol e estudos disponíveis eletronicamente.
A análise foi baseada na classificação dos estudos segundo tipo de produção (dissertação, tese, artigo ou outra natureza), tipo de abordagem (quantitativo ou quantiqualitativo), tipo de estudo referido (transversal, epidemiológico, descritivo, exploratório ou outro), tipo de instrumento de coleta de dados (questionário, roteiro de entrevista, formulário ou outro), tipo de população/ amostra do estudo (trabalhadores do setor de serviço, indústria, agricultura ou outro) e temporalidade (se o estudo foi publicado dentro do período estabelecido, de 1998 a 2008).

\section{Resultados}

O primeiro levantamento resultou no total de 655 pesquisas. Após a leitura dos resumos foram selecionados 33 estudos para análise. Em relação à distribuição dos estudos, segundo as bases de dados consultadas, a maior parte é derivada do Banco de Teses da Capes com 11 (33,3\%), seguido do MEDLINE com $9(27,3 \%)$ e da LILACS com $8(24,2 \%)$. Em relação à metodologia, 29 estudos se basearam no delineamento quantitativo $(87,9 \%)$, enquanto apenas $12,1 \%$ eram quantiqualitativos.

$\mathrm{Na}$ distribuição temporal durante o período compreendido, há concentração após o ano 2004, que agregou 24 publicações, sendo $72,7 \%$ do total. Em relação à natureza da produção, 66,7\% (22) dos estudos selecionados são artigos e 27,3\% (9) são dissertações de mestrado. A quantidade de autores varia de um a mais de três, sendo um autor $(33,3 \%)$ e dois autores $(30,3 \%)$ em grande parte dos estudos, seguido de três $(18,2 \%)$ e mais de três autores $(18,2 \%)$. Os únicos estudos que apresentam apenas um autor são as dissertações de mestrado e as teses de doutorado. Os autores dos estudos foram categorizados em docente $(45,5 \%)$, aluno de pós-graduação $(33,3 \%)$, não especificado $(12,1 \%)$, docente e aluno $(6,1 \%)$ e assistente $(3,0 \%)$.

As pesquisas foram classificadas segundo o desenho metodológico referido, sendo os tipos de estudos mais frequentes os descritivos $(39,4 \%)$, exploratórios $(24,2 \%)$, transversais $(21,2 \%)$ e epidemiológicos $(12,1 \%)$.

A maioria dos trabalhadores participantes das pesquisas não foi especificado quanto à atividade laboral (36,4\%). Enquanto $33,3 \%$ dos trabalhadores petenciam ao setor de serviços, $27,3 \%$ ao setor industrial e 3\% eram trabalhadores rurais. Porém, é possível constatar, através da revisão realizada, reduzido número de pesquisas em indústrias, devido à característica da organização de trabalho, que tende a se constituir em um espaço de trabalho de acesso mais restrito que o setor de serviços.

A técnica de coleta de dados mais utilizada por todos os estudos analisados corresponde ao questionário (90,9\%). Apenas 9,1\% dos estudos selecionados aplicaram, além do questionário, as entrevistas estruturadas. Os instrumentos utilizados em cada estudo, assim como o grupo populacional estudado e o delineamento metodológico estão sintetizados na Figura 1, em ordem cronológica de publicação. 


\begin{tabular}{|c|c|c|c|c|}
\hline $\mathbf{n}$ & $\begin{array}{c}\text { Ano de } \\
\text { publicação }\end{array}$ & $\begin{array}{c}\text { Desenho metodológico } \\
\text { referenciado }\end{array}$ & População ou amostra estudada & Instrumento de coleta de dados \\
\hline 1 & 1998 & Transversal & $\begin{array}{l}\text { Sindicalizados de fábricas de } \\
\text { máquinas pesadas na central } \\
\text { estadual dos EUA }\end{array}$ & $\begin{array}{l}\text { Escala (idade, nível de escolaridade, gênero, etnia, suporte social, } \\
\text { eficácia do Programa de Assistência ao Empregado - EAP, incentivo } \\
\text { ao trabalhador procurar o EAP, consumo de álcool durante a jornada } \\
\text { de trabalho, hábito de beber) }\end{array}$ \\
\hline 2 & 1998 & Epidemiológico & $\begin{array}{l}\text { Trabalhadores do setor industrial } \\
\text { da região metropolitana de } \\
\text { Salvador, BA }\end{array}$ & $\begin{array}{l}\text { Ficha familiar (dados sociodemográficos e familiares); história } \\
\text { migratória e ocupacional (HMO); e questionário de morbidade } \\
\text { psiquiátrica de adultos (QMPA) }\end{array}$ \\
\hline 3 & 1999 & Exploratório & $\begin{array}{l}\text { Trabalhadores de uma organização } \\
\text { de grande porte }\end{array}$ & $\begin{array}{l}\text { Questionário (estresse no trabalho, razões para beber, uso de álcool } \\
\text { e problemas relacionados ao álcool); escala geral de estresse (SIG) }\end{array}$ \\
\hline 4 & 2000 & Exploratório & $\begin{array}{l}\text { Cortadores de cana de uma } \\
\text { agroindústria sucroalcooleira }\end{array}$ & Questionário (dados sociodemográficos) e o Audit \\
\hline 5 & 2001 & Epidemiológico & $\begin{array}{l}\text { Trabalhadores do setor industrial e } \\
\text { de serviço }\end{array}$ & Questionário Employee Health and Performance in the Workplace. \\
\hline 6 & 2001 & Longitudinal & $\begin{array}{l}\text { Trabalhadores do setor industrial e } \\
\text { de serviço }\end{array}$ & $\begin{array}{l}\text { Entrevista (informações demográficas, sobre emprego, educação, } \\
\text { localização geográfica - rural ou urbana, distrito de residência, } \\
\text { estado de saúde geral etc.) }\end{array}$ \\
\hline 7 & 2002 & Descritivo & Trabalhadores & $\begin{array}{l}\text { Questionário (estimar a taxa de prevalência do uso de drogas lícitas } \\
\text { e ilícitas referentes ao último mês e ao último ano; o uso indevido de } \\
\text { substâncias psicoativas e/ou fármacos) }\end{array}$ \\
\hline 8 & 2003 & Exploratório & Trabalhadores do setor industrial & $\begin{array}{l}\text { Questionário (consumo de álcool e drogas, perfil dos trabalhadores, } \\
\text { fator de risco relacionado ao ambiente de trabalho, conhecimentos } \\
\text { acerca do fenômeno álcool e outras drogas, e dados para } \\
\text { implementar a promoção da saúde e a prevenção do uso de álcool } \\
\text { e outras drogas) }\end{array}$ \\
\hline 9 & 2003 & Descritivo & Trabalhadores & Audit e o Cage \\
\hline 10 & 2004 & Exploratório & $\begin{array}{l}\text { Funcionários da Prefeitura do } \\
\text { Campus da Universidade de São } \\
\text { Paulo (USP) }\end{array}$ & $\begin{array}{l}\text { Entrevista clínica estruturada (SCID 2.0); Questionário } \\
\text { sociodemográfico e o Cage }\end{array}$ \\
\hline 11 & 2004 & Descritivo & $\begin{array}{l}\text { Pacientes de centros de } \\
\text { recuperação e membros da } \\
\text { irmandade Alcoólicos Anônimos da } \\
\text { Grande Florianópolis }\end{array}$ & $\begin{array}{l}\text { Escala Inventário dos Fatores de Risco do Trabalho Associados ao } \\
\text { Abuso de Substâncias Psicoativas; e Questionário (características } \\
\text { socioocupacionais, características do uso de substâncias } \\
\text { psicoativas e do tratamento dos participantes) }\end{array}$ \\
\hline 12 & 2004 & Exploratório & Trabalhadores do setor industrial & $\begin{array}{l}\text { Questionário (perfil sociodemográfico e ocupacional dos } \\
\text { trabalhadores, fatores de riscos relacionados ao trabalho, frequência } \\
\text { do uso de álcool, fumo e outras substâncias psicoativas, grau de } \\
\text { satisfação com o trabalho e com o cargo, relações interpessoais } \\
\text { com colegas de trabalho e chefia etc.) }\end{array}$ \\
\hline 13 & 2005 & Descritivo & Mulheres trabalhadoras & $\begin{array}{l}\text { Questionário spciodemográfico e laboral (ESDyL); questionário } \\
\text { TWEAK; tabela de avaliação do consumo de drogas e auestionário } \\
\text { de violência no trabalho }\end{array}$ \\
\hline 14 & 2005 & Descritivo & Estudantes trabalhadores & $\begin{array}{l}\text { Questionário (atividades físicas, nível de estresse, hábitos } \\
\text { alimentares, uso de fumo e bebidas alcoólicas, idade que iniciaram } \\
\text { o consumo e atividades laborais) }\end{array}$ \\
\hline 15 & 2005 & Exploratório & Mulheres trabalhadoras & $\begin{array}{l}\text { Questionário sociodemográfico e de situação de trabalho; } \\
\text { questionário sobre violência no trabalho; questionário TWEAK e } \\
\text { tabela simples de identificação de consumo de drogas ilícitas e } \\
\text { cigarro }\end{array}$ \\
\hline 16 & 2005 & Epidemiológico & Trabalhadores & Questionário Health Behavior Questionnaire (HBQ). \\
\hline 17 & 2005 & Transversal & $\begin{array}{l}\text { Trabalhadores industriais do } \\
\text { Estado de Santa Catarina }\end{array}$ & Questionário estilo de vida e hábitos de lazer/industriários \\
\hline 18 & 2005 & Descritivo & Motoristas de caminhão do Brasil & $\begin{array}{l}\text { Índice da qualidade de sono de Pittsburgh (IQSP); escala de } \\
\text { sonolência de Epworth (ESE); questionário sobre o uso de } \\
\text { medicamentos e drogas; questionário MINI (Mini International } \\
\text { Neuropsychiatric Interview); inventário Beck para depressão (Beck } \\
\text { depression inventory - BDI) }\end{array}$ \\
\hline 19 & 2005 & Descritivo & $\begin{array}{l}\text { Servidores federais: docentes e } \\
\text { técnicos administrativos }\end{array}$ & $\begin{array}{l}\text { Questionário de prontidão para a atividade física (Q-PAF); } \\
\text { questionário (uso regular de medicações, prevalência do uso de } \\
\text { tabaco, consumo de álcool etc.) }\end{array}$ \\
\hline 20 & 2005 & Descritivo & $\begin{array}{l}\text { Estudantes adolescentes } \\
\text { trabalhadores e não trabalhadores } \\
\text { da rede estadual de ensino de } \\
\text { Cuiabá, MT }\end{array}$ & $\begin{array}{l}\text { Questionário para caracterização do consumo de substâncias } \\
\text { psicoativas e o Cage }\end{array}$ \\
\hline 21 & 2006 & Descritivo & Mulheres trabalhadoras & $\begin{array}{l}\text { ESDyL; questionário TWEAK; tabela de avaliação do consumo de } \\
\text { drogas e questionário de violência no trabalho }\end{array}$ \\
\hline 22 & 2006 & Descritivo & $\begin{array}{l}\text { Trabalhadores de saúde do } \\
\text { Programa Saúde da Familia (PSF) }\end{array}$ & $\begin{array}{l}\text { Questionário (características sociodemográficas, check-list sobre as } \\
\text { substâncias consideradas drogas de abuso, assistência de saúde } \\
\text { a usuários de drogas, chek-list com sentimentos dos profissionais } \\
\text { do PSF); escala The Seaman \& Mannello Nurse's Attitudes Toward } \\
\text { Alcohol and Alcoholism Scale }\end{array}$ \\
\hline
\end{tabular}


Figura 1 - continuação

\begin{tabular}{|c|c|c|c|c|}
\hline $\mathbf{n}$ & $\begin{array}{c}\text { Ano de } \\
\text { publicação }\end{array}$ & $\begin{array}{l}\text { Desenho metodológico } \\
\text { referenciado }\end{array}$ & População ou amostra estudada & Instrumento de coleta de dados \\
\hline 23 & 2006 & Transversal & Trabalhadores do setor de serviço & $\begin{array}{l}\text { Índice de capacidade para o trabalho (ICT); escala estresse no } \\
\text { trabalho (EET); Medical Outcomes Study } 36 \text { - Item short form health } \\
\text { survey (SF-36); questionário de Baecke; questionário de tolerância } \\
\text { de Fagerström e Audit }\end{array}$ \\
\hline 24 & 2007 & Descritivo & $\begin{array}{l}\text { Trabalhadores do serviço de coleta } \\
\text { de lixo na periferia da região sul do } \\
\text { município de São Paulo }\end{array}$ & $\begin{array}{l}\text { Questionário: relação do consumo de álcool com a profissão } \\
\text { (QRCAP) }\end{array}$ \\
\hline 25 & 2007 & Exploratório & Mulheres trabalhadoras & $\begin{array}{l}\text { Questionário sobre Violência no Trabalho e guia para entrevista em } \\
\text { profundidade }\end{array}$ \\
\hline 26 & 2007 & Exploratório & Caminhoneiros de estrada & $\begin{array}{l}\text { Questionário (condições de trabalho, tempo de profissão, período } \\
\text { em que costuma trafegar, horas trabalhadas por dia e horas de } \\
\text { descanso, uso de álcool e anfetaminas) }\end{array}$ \\
\hline 27 & 2007 & Descritivo & $\begin{array}{l}\text { Trabalhadores de uma indústria } \\
\text { petroquímica no sul de Veracruz, } \\
\text { México }\end{array}$ & $\begin{array}{l}\text { Ficha de dados pessoais, biológicos e culturais; Audit e o } \\
\text { questionário de afeto relacionado ao consumo de álcool (Carca) }\end{array}$ \\
\hline 28 & 2007 & Transversal & $\begin{array}{l}\text { Adolescentes trabalhadores e não } \\
\text { trabalhadores da rede estadual de } \\
\text { educação básica de Cuiabá, MT }\end{array}$ & $\begin{array}{l}\text { Questionário do IV Levantamento sobre o uso de drogas entre } \\
\text { estudantes de } 1^{\circ} \text { e } 2^{\circ} \text { graus em } 10 \text { capitais brasileiras; HMO e } \\
\text { QMPA }\end{array}$ \\
\hline 29 & 2008 & Transversal & Caminhoneiros de longo percurso & $\begin{array}{l}\text { Questionário Work-Related Safety and Violent Victimization Survey, } \\
\text { escala de conflitos táticos, avaliação de assédio no local de trabalho, } \\
\text { monitoramento da tensão dos caminhoneiros, escala de percepção } \\
\text { do estresse, Cage e características demográficas }\end{array}$ \\
\hline 30 & 2008 & Descritivo & Trabalhadores do setor industrial & Questionário estilo de vida e hábitos de lazer dos industriários \\
\hline 31 & 2008 & Transversal & $\begin{array}{l}\text { Trabalhadores do setor de serviço } \\
\text { - mototaxistas }\end{array}$ & $\begin{array}{l}\text { Questionário (características sociodemográficas, perfil laboral, } \\
\text { comportamento no trânsito, se ingere bebidas alcoólicas, a } \\
\text { frequência da ingestão, a recorrência desse hábito por dia, se dirige } \\
\text { sob o efeito do álcool, bebida que ingere normalmente etc.) }\end{array}$ \\
\hline 32 & 2008 & Transversal & Trabalhadores & $\begin{array}{l}\text { General Health Questionnaire - } 12 \text { (GHQ-12) e Maslach Burnout } \\
\text { Inventory (MBI) }\end{array}$ \\
\hline 33 & 2008 & Epidemiológico & Trabalhadores do setor industrial & $\begin{array}{l}\text { Questionário de Baecke, questionário (hábito de fumar, consumo de } \\
\text { bebidas alcoólicas etc.) }\end{array}$ \\
\hline
\end{tabular}

Figura 1 - Características dos estudos selecionados. Rio de Janeiro, RJ, Brasil, 2010

\section{Discussão}

Entende-se, atualmente, que a flexibilização do trabalho se faz presente na sociedade brasileira e, por isso, muitos trabalhadores encontram no uso de drogas uma forma de escape ${ }^{(7)}$. Os estudos analisados evidenciam que houve preocupação com um grupo significativamente heterogêneo de trabalhadores, mas chama a atenção o fato de que a maioria deles se situa no setor de produção de serviços, fortemente afetado pelo processo de reestruturação produtiva.

Há alguns anos, o uso de drogas no local de trabalho era subestimado pelas empresas. Contudo, observa-se que as empresas atualmente têm se preocupado com o aumento da incidência dessas situações ${ }^{(8)}$. Os resultados da análise das publicações encontradas nesta revisão confirmam que há preocupação crescente com o tema, expressa na concentração de publicações nos últimos anos.

Dessa forma, serão apresentados os instrumentos de coleta de dados utilizados pelos estudos selecionados, a fim de conhecer os instrumentos voltados para a saúde do trabalhador, que abordam a temática álcool e outras drogas.

Foi utilizado o Questionário: relação do consumo de álcool com a profissão (QRCAP), elaborado por uma das pesquisadoras, com o intuito de descrever o consumo de álcool utilizado entre trabalhadores de coleta de lixo. É baseado no teste de identificação de transtorno por uso de álcool (Audit), para identificar e estratificar o alcoolismo ${ }^{(9)}$.
Para comparar a prevalência do uso recente de substâncias psicoativas na população de adolescentes trabalhadores e não trabalhadores, matriculados na rede estadual de educação básica da área urbana de Cuiabá, MT, empregou-se o questionário utilizado no IV Levantamento sobre o uso de drogas entre estudantes de $1^{\circ}$ e $2^{\circ}$ graus em 10 capitais brasileiras ${ }^{(10)}$, o qual foi adaptado $^{(11)}$ à realidade brasileira e testado ${ }^{(12)}$ no contexto cuiabano. $\mathrm{O}$ instrumento de autopreenchimento procurava identificar o uso recente (consumo realizado uma ou mais vezes nos 30 dias que antecederam a aplicação do questionário) de álcool e outras drogas, a idade do primeiro uso, dados sociodemográficos, escolares e de comportamento de saúde, como a prática de esportes. Também outros autores $^{(13)}$ estimaram a prevalência de consumo de álcool e do alcoolismo entre estudantes adolescentes trabalhadores e não trabalhadores da rede estadual de ensino de Cuiabá, MT, usando o mesmo instrumento, sem a presença do professor. Sendo acrescentadas algumas questões sobre alcoolismo, trabalho e família ${ }^{(14)}$. Para detecção do alcoolismo, utilizou-se o teste Cage (Cut-down; Annoyed; Guilty \& Eye-opener), com ponto de corte de duas ou mais respostas afirmativas, o que sugere "rastreamento positivo" para abuso ou dependência de álcool. Nesses estudos, portanto, as variáveis de trabalho foram avaliadas no contexto escolar, com grupo populacional de estudantes, 
o que pode representar limite para a avaliação de questões específicas do trabalho.

Foi testada a hipótese de associação entre migração recente, trabalho na indústria petroquímica e o consumo excessivo de bebidas alcoólicas em trabalhadores do setor industrial da região metropolitana de Salvador, BA, avaliando o papel de variáveis socioeconômicas e demográficas como confundidoras ou modificadoras do efeito. Os dados sociodemográficos e familiares de cada indivíduo foram registrados em uma Ficha Familiar. Os seguintes instrumentos foram utilizados: história migratória e ocupacional (HMO) e questionário de morbidade psiquiátrica de adultos (QMPA), utilizado para identificar os usuários de bebidas alcoólicas. A facilidade de aplicação, nesse caso, surgiu como uma questão metodológica importante, em se tratando de população de escolaridade variável como o adulto trabalhador de indústria ${ }^{(15)}$.

Utilizou-se um questionário anônimo contendo 19 questões de múltipla escolha, abordando condições de trabalho (tempo de profissão, período em que costuma trafegar, horas trabalhadas por dia e horas de descanso) e uso de álcool e anfetaminas (frequência, motivação e local de uso, local de aquisição e se já teve algum acidente na estrada) entre caminhoneiros de estrada. Os caminhoneiros foram abordados em um posto de combustíveis em Passos, MG, quando eram atendidos em uma unidade móvel de saúde ${ }^{(16)}$.

O Cage, novamente, foi utilizado, avaliando-se os indicadores de validade - sensibilidade, especificidade, valor preditivo positivo - entre funcionários da Prefeitura do Campus da Universidade de São Paulo (USP), utilizando a entrevista clínica estruturada (SCID 2.0) para a $4^{\mathrm{a}}$ edição do Manual de Diagnóstico e Estatística das Perturbações Mentais (DSM-IV). Foi aplicado um questionário sociodemográfico seguido do Cage e da SCID 2.0. Os indicadores de validade do Cage foram analisados através dos resultados da SCID 2.0 para abuso e dependência do álcool ${ }^{(17)}$.

O Cage é instrumento utilizado para a detecção de problemas relacionados ao uso do álcool, e, na avaliação dos autores, demonstrou ser instrumento pouco intimidativo, de aplicação fácil e rápida. Tanto o Audit quanto o Cage são ferramentas amplamente utilizadas na avaliação de rastreio de problemas relacionados ao álcool. O Audit foi desenvolvido especificamente para avaliar problemas relacionados ao hábito de beber, enquanto o Cage é instrumento padronizado de rastreio para a detecção de dependência do álcool ${ }^{(17)}$.

Para conhecer de que maneira os fatores pessoais, biológicos, socioculturais e o afeto influenciam no consumo de álcool, entre os trabalhadores de uma indústria petroquímica no sul de Veracruz, México, utilizou-se ficha de dados pessoais, biológicos e culturais, e dois instrumentos: o Audit e o questionário de afeto relacionado ao consumo de álcool (Carca), adaptado de uma escala de adjetivos múltiplos de afeto $^{(18)}$.

A utilidade do Audit e do Cage foi avaliada quanto à identificação do beber em binge ou beber pesado episódico entre trabalhadores altamente qualificados. $\mathrm{O}$ Audit alcançou sensibilidade de $35 \%$ na identificação dos entrevistados que relataram o uso binge de álcool, e especificidade de $98 \%$ em identificar com precisão os respondentes que não relataram o uso binge. Um método mais preciso para avaliar o beber em binge parece estar ligado diretamente ao questionamento do consumo de maior número de bebidas alcoólicas em uma única ocasião $^{(19)}$.

O Audit também foi aplicado a um grupo de cortadores de cana de uma agroindústria sucroalcooleira, nos campos de cultivo da cana-de-açúcar de Ribeirão Preto, a fim de verificar o consumo de álcool e identificar os bebedores de risco. Os dados sociodemográficos foram coletados por um questionário à parte ${ }^{(20)}$.

Também foi avaliada a eficácia do treinamento para prevenção ao uso de substâncias psicoativas no trabalho, entre 450 trabalhadores norte-americanos de diversas categorias, avaliando, primeiramente, mudanças de curto prazo nas atitudes, conhecimento, e variáveis relacionadas ao clima de trabalho, e, em seguida, se o comportamento de procura de ajuda mudou a longo prazo ou 6 meses após o treinamento por meio do Employee Assistance Program (EAP), ou Programa de Assistência ao Empregado. Foi aplicado um questionário intitulado Employee Health and Performance in the Workplace, por uma equipe treinada ${ }^{(21)}$.

$\mathrm{Na}$ perspectiva de avaliar os resultados de um programa de prevenção, outros autores ${ }^{(22)}$ utilizaram o questionário Health Behavior Questionnaire (HBQ), validado pelos pesquisadores em estudos anteriores. $\mathrm{O}$ HBQ mensura o uso de álcool e atitudes; prescrição e consumo de drogas ilícitas; opiniões sobre saúde, bebidas, e uso de drogas; mecanismos e estratégias para alívio do estresse; e utilização dos serviços de saúde e do programa de prevenção. $\mathrm{O}$ abuso de substâncias e problemas de saúde mental foram identificados através da $9^{a}$ Revisão da Classificação Internacional de Doenças, Modificação Clínica (CID-9-CM). Alguns pesquisadores ${ }^{(23)}$, também com foco no EAP, investigaram sobre crença, apoio social e probabilidade de um funcionário procurar o Programa devido a algum problema relacionado ao hábito de beber.

Com o intuito específico de estudar as relações existentes entre a violência no ambiente de trabalho e uso de drogas por mulheres trabalhadoras, foi acordado um conjunto de instrumentos: questionário sobre violência no trabalho, desenvolvido pela Organização Internacional do Trabalho (OIT) e outras organizações, e um guia para entrevista em profundidade, elaborado pelos pesquisadores a fim de orientar a entrevista e obter apenas informações necessárias para uma investigação qualitativa de percepções ${ }^{(24)}$.

$\mathrm{Na}$ perspectiva de que o adoecimento, oriundo das condições de trabalho, afeta o consumo de substâncias, buscou-se determinar a extensão, o padrão e os fatores preditores da morbidade psicológica e o esgotamento (síndrome de Burnout) entre profissionais que abusam de substâncias psicoativas. A morbidade psicológica foi definida com a pontuação no General Health Questionnaire - 12 (GHQ-12). Nesse caso, o pressuposto foi o de que, 
sendo já usuários em situação de abuso, o rastreamento da condição de Burnout evidenciaria a força de associação entre essas duas condiçõos ${ }^{(25)}$.

A preocupação mais recente entre consumo de álcool e drogas e segurança no trânsito evidencia-se no estudo ${ }^{(26)}$ realizado com caminhoneiros de longo percurso. Os participantes preencheram questionário sobre a segurança no trabalho, desenhado pela equipe de pesquisa intitulado Work-Related Safety and Violent Victimization Survey. Também foram utilizadas as seguintes escalas: escala de conflitos táticos, avaliação de assédio no local de trabalho, monitoramento da tensão dos caminhoneiros, escala de percepção do estresse e Cage. Verifica-se, portanto, preocupação em ampliar a inclusão de variáveis por meio de diversos instrumentos.

Buscaram investigar o impacto de problemas relacionados ao alcoolismo sobre o emprego, analisando uma amostra aleatória de homens e mulheres que estavam há mais tempo no trabalho em seis Estados do Sul dos EUA (Alabama, Arkansas, Geórgia, Louisiana, Mississipi e Tennessee). Os participantes da pesquisa foram entrevistados por telefone, discutindo-se uma problemática sobre alcoolismo. A entrevista também incluiu questões relacionadas ao emprego e informações demográficas, como idade, sexo, raça/etnia, estado civil, tamanho da família, educação, localização geográfica (rural ou urbana e distrito de residência), experiência em serviço militar e estado de saúde geral. Foi o único estudo que mencionou entrevista por telefone ${ }^{(27)}$.

A relação entre as situações de estresse no trabalho e razões evasivas para o uso de álcool foi o objeto de uma pesquisa ${ }^{(28)}$. Os participantes responderam perguntas relativas ao estresse no trabalho, razões para beber, uso de álcool e problemas relacionados ao álcool.

A caracterização dos fatores de risco advindos do trabalho, associados ao histórico de dependência ou abuso de substâncias psicoativas, em pacientes de quatro centros de recuperação e de membros da irmandade Alcoólicos Anônimos da Grande Florianópolis, foi realizada pela aplicação de uma escala tipo Likert, inventário dos fatores de risco do trabalho associados ao abuso de substâncias psicoativas, composta de 62 afirmativas ${ }^{(29)}$.

O uso de estimulantes, as queixas relacionadas ao sono e o estado de humor de 300 motoristas de caminhão oriundos de todo o Brasil foram investigados, através da aplicação dos seguintes questionários: índice da qualidade de sono de Pittsburgh (IQSP), escala de sonolência de Epworth (ESE), questionário sobre o uso de medicamentos e drogas, questionário MINI (Mini International Neuropsychiatric Interview), inventário Beck para depressão (Beck Depression Inventory - BDI) ${ }^{(30)}$.

Para monitorar e caracterizar as mudanças na inatividade física de lazer e outros fatores de risco à saúde, em trabalhadores industriais do Estado de Santa Catarina e da Paraíba, utilizou-se o questionário estilo de vida e hábitos de lazer/industriários, já validado no contexto brasileiro $^{(31-32)}$.

Tal questionário foi utilizado em diversas pesquisas do Serviço Social da Indústria (Sesi) em parceria com o Núcleo de Pesquisa em Atividade Física e Saúde (NuPAF) da Universidade Federal de Santa Catarina (UFSC). É dividido em quatro módulos: informações pessoais, características demográficas e socioeconômicas dos indivíduos, indicadores de saúde e comportamentos preventivos (qualidade do sono, nível de estresse, depressão, percepção da vida no lar, no trabalho e no lazer, frequência do consumo de cigarros e de bebidas alcoólicas, controle da pressão arterial, colesterol e diabetes), atividade física e opções de lazer, controle do peso corporal e hábitos alimentares ${ }^{(32)}$.

Para conhecer os possíveis fatores de riscos, relacionados aos hábitos e/ou costumes no ambiente de trabalho, além do uso e abuso de álcool e outras drogas entre trabalhadores de uma empresa, instalada numa área petroquímica, utilizou-se um questionário composto por questões abertas e fechadas, a fim de levantar tais informações e o perfil sociodemográfico e ocupacional dos trabalhadores, o grau de satisfação com o trabalho e com o cargo, relações interpessoais com colegas de trabalho e chefia, conhecimentos acerca do fenômeno álcool e outras drogas, entre outras ${ }^{(4,33)}$.

Aplicaram-se alguns instrumentos em mulheres trabalhadoras que residiam na área metropolitana de Monterrey (México) e na cidade do Rio de Janeiro, a fim de avaliar a proporção de consumo de drogas, identificar os possíveis fatores de riscos sociodemográficos e laborais, e a existência de violência no ambiente laboral. Os instrumentos foram: questionário sociodemográfico e laboral (Encuesta Social Demográfica y Laboral ESDyL), questionário TWEAK - mede o uso e abuso de álcool, tabela de avaliação do consumo de drogas registra a frequência do consumo de drogas ilícitas alguma vez na vida, nos últimos 12 meses, nos últimos 6 meses, no último mês e nos últimos 15 dias e questionário de violência no trabalho - identifica a frequência e os tipos de violência presentes no ambiente de trabalho e suas consequências $^{(5,34)}$.

Novamente estudaram mulheres trabalhadoras que residiam em comunidades de Monterrey, México, de Lima, Peru, e do Rio de Janeiro, Brasil, com o intuito de determinar a proporção de consumo de drogas, os fatores de riscos pessoais e laborais que podem influenciar no consumo de substâncias, a violência laboral e sua relação com as drogas. Para tanto, utilizaram-se os instrumentos supracitados $^{(6)}$.

Realizou-se um estudo na população geral trabalhadora do Chile, através do uso de um questionário baseado no estudo nacional sobre drogas, do Chile. Esse questionário contém um conjunto de perguntas que permite estimar a taxa de prevalência do uso de drogas lícitas e ilícitas, referentes ao último mês e ao último ano, o uso indevido de substâncias psicoativas e/ou fármacos, cujo consumo se dá sem receita médica ${ }^{(35)}$.

Os indicadores gerais de saúde, dados demográficos e socioeconômicos foram investigados em um grupo de servidores federais. Foi aplicado o questionário de prontidão para a atividade física (Q-PAF), adaptado do Ministério da Saúde do Canadá, para avaliar o estado 
de saúde atual, uso regular de medicações, prevalência do uso de tabaco, consumo de álcool, doenças dos diversos aparelhos e sistemas, histórico familiar e outras informações ${ }^{(36)}$.

Investigaram-se estudantes trabalhadores dos cursos de eletromecânica e têxtil do Centro Federal de Educação Tecnológica de Santa Catarina (CEFET/SC). Buscouse conhecer as informações sociodemográficas, estilo de vida, atividades laborais e físicas, nível de estresse, hábitos alimentares, comportamento de risco (uso de fumo e bebidas alcoólicas de forma experimental e regular, bem como a idade do início do uso), comportamento preventivo (identificar o uso de drogas e do cinto de segurança) ${ }^{(37)}$.

Os trabalhadores de uma empresa privada do setor elétrico do Estado de São Paulo foram analisados por meio de instrumentos autoaplicáveis, como: o índice de capacidade para o trabalho (ICT) - possibilitando avaliar e detectar precocemente alterações e subsidiando a escolha das medidas preventivas, escala estresse no trabalho (EET) - permite avaliar 23 diferentes aspectos do trabalho, a partir da percepção do próprio trabalhador e obter medida geral do estresse no trabalho, Medical Outcomes Study 36 - Item short form health survey (SF-36) - é um questionário genérico de avaliação de saúde, composto por 36 itens, não sendo específico para uma determinada idade, doença ou grupo de tratamento, questionário de Baecke - investiga a atividade física habitual dos últimos 12 meses, questionário de tolerância de Fagerström - tem a finalidade de identificar e medir a dependência nicotínica. Tal questionário é composto por três questões que avaliam fumo matinal, considerando-o como indicador da síndrome de abstinência, e por três questões que avaliam o consumo de cigarro. $\mathrm{O}$ escore final varia de 0 a 10 pontos, que pode ser categorizado em quatro graus de dependência, e o Audit ${ }^{(38)}$.

Os executivos de duas unidades fabris, de São Caetano do Sul e de São José dos Campos, foram avaliados quanto à atividade física habitual e às condições de saúde. Para avaliar a atividade física habitual nos últimos 12 meses aplicou-se o questionário de Baecke. Em relação ao tabagismo, os autores criaram quatro perguntas sobre o hábito de fumar, baseado nos questionários da The Third National Health and Nutrition Examination Survey NHANES III ${ }^{(39)}$ e do Behavioral risk factor surveillance system survey questionnaire - BRFSS ${ }^{(40)}$, ambos inquéritos de base populacional, realizados nos EUA. Na avaliação do consumo de bebidas alcoólicas, uma pergunta sobre o uso atual de álcool foi criada baseada no módulo de consumo do Instituto Nacional de Câncer - Inca ${ }^{(41)}$ e do BRFSS $^{(40)}$. Esses instrumentos avaliam a proporção de pessoas que consomem bebidas alcoólicas (consumo atual - pelo menos uma dose nos últimos 30 dias) e estima o percentual de indivíduos que normalmente o fazem acima das recomendações (consumo de risco) ${ }^{(42)}$.

$\mathrm{Na}$ investigação do comportamento preventivo e de risco para o acidente de trânsito, referido por mototaxistas regulamentados em Fortaleza, Ceará, utilizou-se um questionário autoaplicável, composto por 41 perguntas que versavam sobre as características sociodemográficas, o perfil laboral dos mototaxistas, o comportamento no trânsito em relação à moto (se conduz mais de um passageiro, se atende o telefone celular no trânsito, se ingere bebidas alcoólicas, a frequência da ingestão, a recorrência desse hábito por dia, se dirige sob efeito do álcool, a bebida que ingere normalmente etc. $)^{(43)}$.

As atitudes dos profissionais do Programa de Saúde da Família (PSF) frente ao uso, abuso e dependência de drogas foram investigadas através da aplicação de um questionário nas equipes do PSF do município de Araçatuba, SP. O questionário foi desenvolvido e estruturado para ser aplicado individualmente: características sociodemográficas; check-list sobre as substâncias que são consideradas drogas de abuso pelos profissionais do PSF, assistência de saúde aos usuários de drogas no PSF, chek-list referente a sentimentos dos profissionais do PSF ao lidarem com uma clientela com problemas relacionados ao uso de drogas e a escala The Seaman \& Mannello Nurse's Attitudes Toward Alcohol and Alcoholism Scale, que é composta por 30 avaliações sobre crenças, atitudes e cuidados prestados pelos enfermeiros em relação ao alcoolismo ${ }^{(44)}$.

Os questionários são menos custosos, exigem menos tempo e esforço para administrar, e têm como vantagem a possibilidade do anonimato, que pode ser crucial na obtenção de informações sobre comportamentos ilegais ou desviados, ou sobre características embaraçosas ${ }^{(45)}$. Quando se investiga o padrão de consumo de álcool e outras drogas entre trabalhadores, isso pode ser de especial interesse metodológico, já que o trabalhador tende a sentirse julgado e intimidado pelo tema abordado, imaginando que eventuais sanções trabalhistas possam decorrer dos resultados do estudo. $\mathrm{Na}$ entrevista face a face, o índice de resposta tende a ser mais alto, pois os respondentes têm menos probabilidade de ignorar um questionário, mas correm o risco de parcialidade do entrevistador.

\section{Conclusões}

Como síntese dos resultados, tem-se que o questionário foi o instrumento de coleta de dados mais utilizado pelos estudos, e se observou busca pela mensuração de características sociodemográficas, laborais e psicossociais para associação aos índices de prevalência de consumo e padrão de uso de álcool e drogas.

O Cage e o Audit foram os mais destacados, principalmente, por serem ferramentas utilizadas na avaliação de rastreio de problemas relacionados ao álcool. No que se refere ao uso de escalas e questionários sobre variáveis de trabalho, essas foram, na sua maioria, obtidas a partir de aspectos subjetivados pelo trabalhador, tais como as escalas de Burnout e estresse. No entanto, nem o Audit e nem o Cage alcançaram a sensibilidade adequada, bem como a especificidade, como instrumentos de rastreio de avaliação do beber em binge.

A avaliação de condições objetivas de trabalho não surge como dimensão contemplada nos estudos. Em especial, situações relativas à organização do trabalho, que sofrem mudanças importantes no contexto de 
reestruturação produtiva atual, com consequente aumento de intensidade e ritmo, podem se constituir em aspectos a serem investigados em estudos posteriores, na busca de maior compreensão sobre um fenômeno complexo como o uso e abuso de substâncias e sua relação com o mundo do trabalho.

\section{Referências}

1. Naciones Unidas Oficina Contra La Droga y El Delito (2003). Tendencias Mundiales de las Drogas Ilícitas. Nueva York [Internet]; 2003 [acesso 10 mar 2010]. Disponível em: http://www.unodc.org/pdf/trends2003_www_S.pdf.

2. Laranjeira R, Pinsky I, Zaleski M, Caetano R. I Levantamento Nacional sobre os padrões de consumo de álcool na população brasileira. Brasília: Secretaria Nacional Antidrogas [online]; 2007 [acesso 25 nov 2007]. Disponível: http://www.obid.senad.gov.br/ http://www. senad.gov.br.

3. Magallón TJC, Robazzi MLCC. Consumo de Alcohol en Trabajadores de Una Industria en Monterrey, México. Rev. Latino-Am. Enfermagem. 2005;13(esp):819-26.

4. Carrillo LPL, Mauro MYC. Uso e abuso de álcool e outras drogas: ações de promoção e prevenção no trabalho. Rev Enferm UERJ. 2003;11(1):25-33.

5. David HMSL, Caufield C. Mudando o foco: um estudo exploratório sobre uso de drogas e violência no trabalho entre mulheres das classes populares da cidade do Rio de Janeiro, Brasil. Rev. Latino-Am. Enfermagem. 2005;13(esp:1148-54.

6. Alonso MMC, Musayon FYO, David HMSL, Gómez MVM. Consumo de drogas y violencia laboral en mujeres que trabajan, un estúdio multicéntrico: México, Peru, Brasil. Rev Rev. Latino-Am. Enfermagem. 2006;14(2):155-62.

7. Abramides MBC, Cabral MSR. Regime de Acumulação Flexível e Saúde do Trabalhador. São Paulo Perspect. 2003;17(1):3-10.

8. Roberto CS, Conte M, Mayer RTR, Torossian SD, Vianna TR. Drogas e trabalho: uma proposta de intervenção nas organizações. Psicol Cienc Prof [periodico na Internet]. 2002 [acesso 20nov 2007]; 22(1):18-29. Disponível em: http://pepsic. bvs-psi.org.br/scielo.php?script=sci_arttext\&pid=S141498932002000100004\&lng-pt\&nrm=iso.

9. Mabuchi AS, Oliveira DF, Lima MP, Conceição MB, Fernandes H. Uso de Bebidas Alcoólicas por Trabalhadores do Serviço de Coleta de Lixo. Rev. LatinoAm. Enfermagem. 2007;15(3):446-52.

10. Souza DPO, Silveira Filho DX. Uso recente de álcool, tabaco e outras drogas entre estudantes adolescentes trabalhadores e não trabalhadores. Rev Bras Epidemiol. 2007;10(2):276-87.

11. Galduróz JCF, Nodo AR, Carlini EA. IV levantamento sobre o uso de drogas entre estudantes de $1^{\circ}$ e $2^{\circ}$ graus em 10 capitais brasileiras. São Paulo: Centro Brasileiro de Informações sobre Drogas Psicotrópicas - CEBRID, Departamento de Psicobiologia da Universidade Federal de São Paulo; 1997. In: Souza DPO, Silveira DX Filho. Uso recente de álcool, tabaco e outras drogas entre estudantes adolescentes trabalhadores e não trabalhadores. Rev Bras Epidemiol. 2007;10(2):276-87.
12. Souza DPO, Martins DTO. O perfil epidemiológico do uso de drogas entre estudantes de $1^{\circ}$ e $2^{\circ}$ graus da rede estadual de ensino de Cuiabá, 1995. Cad Saúde Pública. 1998;14(2):391-400. In: Souza DPO, Silveira DX Filho. Uso recente de álcool, tabaco e outras drogas entre estudantes adolescentes trabalhadores e não trabalhadores. Rev Bras Epidemiol. 2007;10(2):276-87.

13. Souza DPO, Areco KN, Silveira Filho DX. Álcool e alcoolismo entre adolescentes da rede estadual de ensino de Cuiabá, Mato Grosso. Rev Saúde Pública. [periódico na Internet]. 2005 [acesso 20 jun 2009]; 39(4):585-92. Disponível em: http://www.fsp.usp.br/rsp.

14. Smart RG, Hughes DPH, Johnston LD, Anumonye A, Khant U, Medina-Mora ME, et al. A methodology for students drug-use surveys. Geneva: World Health Organization; 1980. (WHO - Offset Publication, 50). In: Souza DPO, Areco KN, Silveira DX Filho. Álcool e alcoolismo entre adolescentes da rede estadual de ensino de Cuiabá, Mato Grosso. Rev Saúde Pública. [periódico na Internet]. 2005 [acesso20 jun 2009]; 39(4):585-92. Disponível em: http://www.fsp.usp.br/rsp.

15. Santana VS, Khoury M, Andrade C, Novato A, Almeida Filho N. Migração, trabalho na indústria petroquímica e consumo de bebidas alcoólicas. Rev Bras Epidemiol. 1998;1(2):149-60.

16. Nascimento EC, Nascimento E, Silva JP. Uso de álcool e anfetaminas entre caminhoneiros de estrada. Rev Saúde Pública. 2007;41(2): 90-3.

17. Amaral RA, Malbergier A. Avaliação de instrumento de detecção de problemas relacionados ao uso do álcool (CAGE) entre trabalhadores da Prefeitura do Campus da Universidade de São Paulo (USP) - Campus Capital. Rev Bras Pisquiatr. 2004;26(3) 156-63.

18. Ramírez LC, Castillo MMA. Afecto y Consumo de Alcohol en Trabajadores de la Industria Petroquímica del Sur de Veracruz, México. SMAD - Rev Eletrônica Saúde Mental Álcool e Drogas [periódico na Internet]. 2007 [acesso 20 jun 2009]; 3(1): [art. 01]. Disponível em: http:// www2.eerp.usp.br/resmad/artigos.asp.

19. Matano RA, Koopman C, Wanat SF, Whitsell SD, Borggrefe A, Westrup D. Assessment of binge drinking of alcohol in highly educated employees. Addict Behav. 2003;28:1299-310.

20. Gonçalves JRL. O Uso de Bebidas Alcoólicas entre os Cortadores de Cana de Uma Agroindústria Sucroalcooleira [dissertação]. Ribeirão Preto (SP): Escola de Enfermagem de Riberão Preto da Universidade de São Paulo; 2000.

21. Bennett JB, Lehman WEK. Workplace Substance Abuse Prevention and Help Seeking: Comparing TeamOriented and Informational Training. J Occup Health Psychol. 2001;6(3):243-54.

22. Deitz D, Cook R, Hersch R. Workplace Health Promotion and Utilization of Health Services. J Behav Health Serv Res. 2005; 32(3): 306-19.

23. Delaney W, Grube JW, Ames GM. Predicting likelihood of seeking help through the employee assistance program among salaried and union hourly employees. Addiction. 1998;93(3):399-410.

24. Musayón Oblitas FY, Caufield C. Workplace violence and drug use in women workers in a Peruvian Barrio. Int Nurs Rev. 2007;54:339-45. 
25. Oyefeso A, Clancy C, Farmer R. Prevalence and associated factors in burnout and psychological morbidity among substance misuse professionals. BMC Health Serv Res. 2008;8(39):1-9.

26. Gay Anderson D, Riley P. Determining Standards of Care for Substance Abuse and Alcohol Use in Long-Haul Truck Drivers. Nurs Clin North Am. 2008;43:357-65.

27. Feng W, Zhou W, Butler JS, Booth BM, French MT. The impact of problem drinking on employment. Health Econ. 2001; 10: 509-21.

28. Grunberg L, Moore S, Anderson-Connolly R, Greenberg E. Work Stress and Self-Reported Alcohol Use: The Moderating Role of Escapist Reasons for Drinking. J Occup Health Psychol. 1999;4(1):29-36.

29. Baumer CMWC. Fatores de risco do trabalho associados ao histórico de dependência ou abuso de substâncias psicoativas [dissertação]. Florianópolis: Universidade Federal de Santa Catarina; 2004.

30. Pinho RSN. Uso de estimulantes, queixas do sono e estado de humor em motoristas profissionais de caminhões [dissertação]. Fortaleza: Faculdade de Farmácia, Odontologia e Enfermagem da Universidade Federal do Ceará; 2005.

31. Fonseca SA. Inatividade física no lazer e outros fatores de risco à saúde em industriários catarinenses, 1999 e 2004 [dissertação]. Florianópolis: Universidade Federal de Santa Catarina; 2005.

32. Araújo VC. Prevalência e fatores associados à inatividade física em trabalhadores da indústria da Paraíba [dissertação]. Florianópolis: Universidade Federal de Santa Catarina; 2008.

33. Carrillo PLL, Mauro MYC. O trabalho como fator de risco ou fator de proteção para o consumo de álcool e outras drogas. Texto Contexto Enferm. 2004;13(2):217-25.

34. Alonso MMC, Caufield C, Gómez MVM. Consumo de drogas y violencia laboral en mujeres trabajadoras de Monterrey, N. L., México. Rev. Latino-Am. Enfermagem. 2005;13(esp):1155-63.

35. Araneda JCF, Cumsille FG. Uso de drogas en la población laboral de Chile. Bol Cient Asoc Chil Segur. 2002;4(8):13-24.

36. Oliveira ESA. Atividade física habitual e outros comportamentos relacionados à saúde dos servidores da Universidade Federal de Santa Catarina: tendência secular 1994-2004 [dissertação]. Florianópolis: Universidade Federal de Santa Catarina; 2005.

37. Bianchetti LA. Estilo de vida de estudantes trabalhadores do Centro Federal de Educação Tecnológica de Santa Catarina-Unidade Jaraguá do sul (CEFET/SCJS) [dissertação]. Florianópolis: Universidade Federal de Santa Catarina; 2005.
38. Martinez MC. Estudo dos fatores associados à capacidade para o trabalho em trabalhadores do Setor Elétrico [tese]. São Paulo: Universidade de São Paulo; 2006.

39. The Third National Health and Nutrition Examination Survey - NHANES III. Centers for Disease Control and Prevention. [acesso $28 \mathrm{dez}$ 2007] Disponível em: $\quad$ http://www.cdc.gov/nchs/about/major/nhanes/ nhanes2005-2006/questexam05-06.htm.. In: Santos LA. Atividade física e morbidade cardiovascular referidas pelos gerentes e diretores de uma indústria automobilística: influência de um programa de condicionamento físico supervisionado [tese]. São Paulo: Universidade de São Paulo; 2008.

40. Behavioral risk factor surveillance system survey questionnaire - BRFSS. Centers for Disease Control and Prevention. [acesso $28 \mathrm{dez}$ 2007] Disponível em: http:// www.cdc.gov/brfss/questionnaires/pdf-ques/2007brfss. pdf. In: Santos LA. Atividade física e morbidade cardiovascular referidas pelos gerentes e diretores de uma indústria automobilística: influência de um programa de condicionamento físico supervisionado [tese]. São Paulo: Universidade de São Paulo; 2008.

41. Ministério da Saúde. Instituto Nacional de Câncer (BR). Inquérito Domiciliar sobre Comportamentos de Risco e Morbidade Referida de Doenças e Agravos não Transmissíveis em 15 capitais brasileiras e no Distrito Federal. [acesso 28 dez 2007] Disponível em: http://www. inca.gov.br/inquerito/docs/completa.pdf. In: Santos LA. Atividade física e morbidade cardiovascular referidas pelos gerentes e diretores de uma indústria automobilística: influência de um programa de condicionamento físico supervisionado [tese]. São Paulo: Universidade de São Paulo; 2008.

42. Santos LA. Atividade física e morbidade cardiovascular referidas pelos gerentes e diretores de uma indústria automobilística: influência de um programa de condicionamento físico supervisionado [tese]. São Paulo: Universidade de São Paulo; 2008.

43. Lira SVG. Comportamento preventivo e de risco no trânsito, referido por mototaxistas regulamentados em Fortaleza (CE) [dissertação]. Fortaleza: Universidade de Fortaleza; 2008.

44. Barros MA. Os profissionais do Programa Saúde da Família frente ao uso, abuso e dependência de drogas [dissertação]. São Paulo: Universidade de São Paulo; 2006. 45. Polit DF, Beck CT, Hungler BP. Fundamentos de Pesquisa em Enfermagem: métodos, avaliação e utilização. $5^{\mathrm{a}}$ ed. Porto Alegre: Artmed; 2004.

\section{Como citar este artigo:}

Rocha PR, David HMSL. Questionários sobre o uso de álcool e drogas entre trabalhadores: revisão da literatura. SMAD, Rev. Eletrônica Saúde Mental Álcool Drog. (Ed. port.). maio-ago. 2011 [acesso: Disponível em: ]; $7(2): 107-16$.

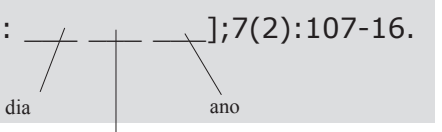

mês abreviado com ponto 